\title{
Commutable Matrix-Valued Functions and Operator-Valued Functions
}

\author{
Abdelaziz Maouche ${ }^{1^{\star}}$
}

Abstract
A simple expression is established for an analytic commutable matrix-valued function
two by two functional commutative matrices is proven. Finally, a family of analy
a Hilbert space, which commute with their derivatives, is shown to be functionally
Keywords: Analytic matrix-valued function, Commutable matrices, Eigenvalue,
function, Resolvent, Riesz projection, Spectrum
2010 AMS: Primary 47B15, Secondary 47A55
${ }^{1}$ Department of Mathematics, Faculty of Science, Sultan Qaboos University, Oman
${ }^{*}$ Corresponding author: maouche@squ.edu.om
Received: 28 June 2020, Accepted: 18 December 2020, Available online: 22 December 2020

\section{Introduction}

The theory of analytic perturbation is historically the first subject in perturbation theory. It is mainly concerned with the behaviour of isolated eigenvalues and eigenvectors (or eigenprojections) of an operator depending on a parameter holomorphically. Once the notion of holomorphic dependence of an (in general unbounded) operator on a parameter is introduced, it is rather straightforward to show that the results obtained in the finite-dimensional case can be extended, at least for isolated eigenvalues, without essential modification. This is exactly what we aim to achieve in our present work. Indeed, in the first part of this paper we study matrix-valued analytic functions which commute with their derivatives. We recall the main theorems obtained by many authors such as Schwerdtfeger [15], Dieudonné [4], Goff [7] and Evard [6]. First we comment some of these results by adding some remarks and examples to see if there is a possibility to extend some of them or not. We recall that Theorem 1 in [4] is an extension of the main theorem of [15] from analytic matrix functions to continuously differentiable functions. Then we state J. Claude Evard's theorem on continuously differentiable and diagonalizable matrices with a constant number of eigenvalues [6]. Our first result, namely Theorem 3.14, extends this theorem to the case of an analytic family of matrices which are not supposed to be diagonalizable. Then in after a preparatory lemma we give a very simple expression of a functionally commutable analytic matrix-valued function on a real interval and in Theorem 3.17, we study functional commutativity in the case of two by two matrices.

The second part of the paper deals with the extension of some of the above results from the case of matrix-valued analytic functions to the infinite-dimensional situation of analytic operator-valued functions on a Hilbert space. We first recall our main result in the case of analytic compact and self-adjoint oprator functions on a Hilbert space ([12], Theorem 2). Then we show that this result can be extended to the more general situation of analytic normal compact operator-valued functions on a Hilbert space. Indeed, the proof of Theorem 2 in[12] is based on the Spectral Theorem for Compact self-adjoint operators, but this spectral representation is still valid for normal compact operators on a Hilbert space as established recently in Theorem 3.3 of [11]. We end this paper by two open questions worth studying for their importance in applications to problems on 
differential equations and other topics as can be seen from the historical overview given in [6] about problems related to functional commutativity in general.

\section{Preliminaries}

Our interest in Section 3 focuses on analytic matrix-valued functions, these are functions that can be represented by Taylor series at some point of a real interval $I$. The main point is whether these matrices are diagonalizable or not, that is everything boils down to the nature of the eigenvalues of these matrices, in other words, to their spectrum. We start with Example 1, which exhibits some features of general analytic matrices through a simple two by two matrix. A neat idea of what causes difficulties in the problem of functional commutativity is clearly highlighted by this example. To overcome such situations involving singularities, we present a theorem from [14] dealing with diagonalizability of analytic hermitian matrix functions on a real interval (Theorem 4). Then we move to the core of the present paper, that is functional commutativity of analytic function matrices commuting with their derivatives. The first theorem in this direction was due to Shwertdfeger [15]. It shows that every analytic diagonalizable matrix function is functionally commutative. This is followed by a more general result, which was generalized by Evard [6], who showed that any continuously differentiable family of diagonalizable matrices with a constant number of eigenvalues is functionally commutative. At this point, one can ask if this theorem can be extended, in other words can we drop the condition on the constancy number of eigenvalues. The answer is affirmative, of course not on all the interval $I$, but on the interval minus an exceptional set. Here we make use of a powerful theorem on the scarcity of elements with finite spectrum in Banach algebras ([1], Theorem 3.4.25). This new result is presented in Theorem 3.13. Finally, we prove that matrices which are functionally commutative can be expressed in a very simple form, namely as linear combination of constant matrices with coefficients given by analytic scalar functions as done in Lemma 1 . We close the section by a nice characterization of functionally commutative two by two matrices. This is presented in Theorem 3.17. Then we turn our attention to the case of compact operators on a separable Hilbert space. First, we recall that analytic hermitian function matrices which commute with their derivative on some real interval $I$, i.e, $\left[A(t), A^{\prime}(t)\right]=0$ for all $t \in I$, where we use the bracket notation $\left[A(t), A^{\prime}(t)\right]=0$ instead of $A(t) A^{\prime}(t)=A(t) A(t)$, were studied in [7]. As a main result, it was shown that these matrices are functionally commutative on $I$, i.e., $[A(s), A(t)]=0$ for all $s, t \in I$ ([7], Theorem 3.6). Subsequently in [6], the study of the nonlinear differential equation $\left[A(t), \frac{d A(t))}{d t}\right]=0, t \in \Lambda$, where $\Lambda$ is an open interval in $\mathbb{R}$ and $A$ is a differentiable map from $\Lambda$ into the $\mathbb{C}$-Banach space $M_{n}$ of all $n \times n$ matrices $\left(\alpha_{i, j}\right)$, with $\alpha_{i, j} \in \mathbb{C}$ for $i, j \in\{1, \cdots, n\}$, led the author to consider the more general problem where $\Lambda$ is an open connected subset of a Banach space on $\mathbb{R}$ or $\mathbb{C}$. Thus obtaining Theorem 4.3 in [6], which generalizes the main theorem of [7]. Moreover, [6] contains both a comprehensive historical summary on the motivations behind the problem on matrix functions commuting with their derivatives and further paths of investigations such as the one of interest to us. Indeed this is illustrated by our main result, Theorem 2 in [12], which extends the finite dimensional result of Goff [7] to the infinite-dimensional situation of compact self-adjoint operators on a Hilbert space. In section 4, we study analytic families of normal compact operators, on a complex Hilbert space, which commute with their derivative on some real interval $I$. Our main result establishes that these operators must be functionally commutative on $I$, that is, $[A(s), A(t)]=0$ for all $s, t \in I$, thus extending the main result of [12].

To make the paper as self-contained as possible, we include the proofs of some well known theorems. We denote by $\mathscr{B}(\mathscr{H})$ the Banach algebra of bounded operators on the Hilbert space $\mathscr{H}$.

\subsection{Adjoint of bounded operators in Hilbert spaces}

Proposition 2.1. Let $T \in \mathscr{B}(\mathscr{H})$ be a bounded operator. For all $x \in \mathscr{H}$, there exists a unique $T^{*} x \in \mathscr{H}$ such that

$$
\forall y \in \mathscr{H},\left\langle T y, x>=<y, T^{*} x>\text { and }<x, T y>=<T^{*} x, y>.\right.
$$

The application $T^{*}: \mathscr{H} \longrightarrow \mathscr{H}$ is a bounded operator called the adjoint of $T$.

An important definition can be given now.

Definition 2.2. Let $T \in \mathscr{B}(\mathscr{H})$, then:

- $T$ is self-adjoint if $T^{*}=T$;

- $T$ is normal if $T^{*} T=T T^{*}$, i.e. it commutes with its adjoint;

- $T$ is unitary if $T T^{*}=T^{*} T=I_{\mathscr{H}}$, where $I_{\mathscr{H}}$ is the identity operator on $\mathscr{H}$, i.e. $T$ is invertible and its inverse $T^{-1}=T^{*}$.

Note that any self-adjoint operator is normal, any unitary operator is normal. 


\subsection{The resolvent and spectrum of a bounded operator}

Let $E$ be a complex Banach space and let $T \in \mathscr{B}(E)$. The spectrum of $T$ can be seen as the generalization in infinite dimension of the notion of eigenvalues in finite dimension. Let us start by the complementary set.

Definition 2.3. Let $T \in \mathscr{B}(E)$. The resolvent set of $T$ is the set

$$
\rho(T):=\{\lambda \in \mathbb{C}: \lambda-T \text { is invertible }\} .
$$

The resolvent is the map

$$
\rho(T) \longrightarrow \mathscr{B}(E): \lambda \longmapsto R_{\lambda}(T)=(\lambda-T)^{-1} .
$$

By $\lambda-T$ we mean $\lambda I_{E}-T$, where $I_{E}$ is the identity operator of $E$. Note that, as a consequence of the closed Graph theorem, if $T$ is bounded and invertible, then its inverse is automatically bounded.

Definition 2.4. Let $T \in \mathscr{B}(E)$. The spectrum of $T$ is

$$
\operatorname{Sp}(\mathrm{T}):=\mathbb{C} \backslash \rho(\mathrm{T})=\{\lambda \in \mathbb{C}: \lambda-\mathrm{T} \text { is invertible }\} .
$$

Definition 2.5. An eigenvalue of $T$ is a number $\lambda \in \mathbb{C}$ such that $\operatorname{ker}(T-\lambda) \neq\{0\}$. The set formed by the eigenvalues, denoted by $\mathrm{Sp}_{\mathrm{p}}(\mathrm{T})$, is called the point spectrum.

We have $\operatorname{Sp}_{\mathrm{p}}(\mathrm{T}) \subset \mathrm{Sp}(\mathrm{T})$.

Proposition 2.6. In finite dimension, the spectrum and the point spectrum coincide.

Proof. In finite dimension, the operator $T-z$ is injective if and only if it is surjective, whereas the continuity is always guaranteed.

Theorem 2.7. Let $T \in \mathscr{B}(E)$ where $E$ is a Banach space. Then $\rho(T)$ is an open subset of $\mathbb{C}$.

\subsection{Digression: The notion of analyticity}

Let $\Omega$ be a non-empty subset in $\mathbb{C}$. We say that $f: \Omega \longrightarrow E$ is holomorphic when, for all $z \in \Omega$ the limit

$$
\lim _{w \longrightarrow z} \frac{f(w)-f(z)}{w-z}
$$

exists in the norm of $E$. It is denoted $f^{\prime}(z)$.

Proposition 2.8. Let $f: \Omega \longrightarrow E$. Then $f$ is holomorphic if and only if it is weakly holomorphic, i.e. $\ell \circ f$ is holomorphic on $\Omega$ for all $\ell \in E^{\prime}$ (i.e. the dual space of $\left.E\right)$.

Proof. See Chapter 1 in [8].

Corollary 2.9. Let $f: \mathbb{C} \longrightarrow E$ be holomorphic. If $f$ is bounded, then it is constant.

Proof. Assume that we can find $z_{0} \in \mathbb{C}$ and $z_{1} \in \mathbb{C}$ such that $f\left(z_{0}\right) \neq f\left(z_{1}\right)$. Then by the Hahn-Banach theorem, there exists some $\ell \in E^{\prime}$ such that $\ell \circ f\left(z_{0}\right) \neq \ell \circ f\left(z_{1}\right)$. By the classical Liouville's theorem, it must be constant. This is a contradiction.

The mathematical theory of Banach space valued analytic functions parallels the classical theory of analytic functions as is well presented in Chapter I of [8]. For example, if $\gamma$ is a closed path in a simply connected domain $\Omega$, then

$$
\oint_{\gamma} f(z) d z=0 .
$$

(The integral defined in the usual way by the norm convergent Riemann sums.) To prove (2.1), note that for $\ell \in E^{\prime}$,

$$
\ell\left(\oint_{\gamma} f(z) d z\right)=\oint_{\gamma} \ell(f(z)), d z=0 .
$$

Since $E^{\prime}$ separates points in $E,(2.1)$ holds. 
Starting with (2.1) one obtains in the usual way the Cauchy integral formula

$$
\frac{1}{2 \pi i} \oint_{|w-z|<r} \frac{f(w)}{w-z} d w=f(z) .
$$

Starting with the Cauchy integral formula one proves that for $w \in \Omega$,

$$
f(z)=\Sigma_{n=0}^{\infty} a_{n}(z-w)^{n}
$$

where $a_{n} \in E$. The power series converges and the representation holds in the largest open disk centered at $w$ and contained in $\Omega$. An excellent reference on this topic is [8].

As an example, the resolvent map is analytic.

Theorem 2.10. Let $T \in \mathscr{B}(E)$ where $E$ is a Banach space. Then the map $\lambda \mapsto R_{\lambda}(T)$ is a holomorphic function from $\rho(T)$ into $\mathscr{B}(E)$.

Another useful application of holomorphy is in obtaining the so called Riesz projections.

Proposition 2.11. Let us consider a bounded operator $T \in \mathscr{B}(E)$ where $E$ is a Banach space and $\lambda \in \mathbb{C}$ as an isolated element of $\mathrm{Sp}(\mathrm{T})$. Let $\Gamma_{\lambda} \subset \rho(T)$ be a contour that enlaces only $\lambda$ as element of the spectrum of $T$. Define

$$
P_{\lambda}:=\frac{1}{2 \pi i} \oint_{\Gamma_{\lambda}}(z-T)^{-1} d z
$$

The bounded operator $P_{\lambda}: E \longrightarrow \operatorname{Dom}(\mathrm{T}) \subset \mathrm{F}$ commutes with $T$ and does not depend on the choice of $\Gamma_{\lambda}$. The operator $P_{\lambda}$ is a projection and

$$
P_{\lambda}-I d=\frac{1}{2 \pi i} \oint_{\Gamma_{\lambda}}(\zeta-\lambda)^{-1}(T-\lambda)(\zeta-T)^{-1} d \zeta
$$

It is appropriate to recall at this point that most of the results on analytic matrix functions that we use in the next section can be obtained by using finite-dimensional operators on a Hilbert space. We have already seen above that the spectrum of an operator in finite dimension coincides with the point spectrum. Next, we look at the notion of finite-algebraic multiplicity. For the proofs of the next results and more details see [9].

Proposition 2.12. Assume that the Hilbert space $\mathscr{H}$ is of finite dimension. Fix $T$ in $\mathscr{B}(\mathscr{H})$ and let $\lambda \in \operatorname{Sp}(\mathrm{T})$. Then, $\lambda$ is an eigenvalue. If $\Gamma_{\lambda}$ is a contour enlacing only $\lambda$, then $P_{\lambda}$ is the projection on the algebraic eigenspace associated with $\lambda$.

Proof. It is well known that $\mathscr{H}$ can be written as a sum of the eigenspaces $\mathscr{H}_{j}$ associated with the distinct eigenvalues of $T$. The eigenspaces $\mathscr{H}_{j}$ are stable under $T$. We can assume that $\mathscr{H}_{1}$ is associated with $\lambda$. There exists a basis of $\mathscr{H}$ such that the matrix of $T$ is block diagonal $\left(T_{1}, \cdots, T_{k}\right)$ where the $T_{j}$ is the (upper triangular) matrix of $T_{\mathscr{H}}$. In this adapted basis, the matrix of $P_{\lambda}$ is block diagonal $\left(P_{\lambda, 1}, \cdots, P_{\lambda, k}\right)$. By holomorphy, we have $P_{\lambda, j}=0$ when $j \neq 1$. To simplify, assume that $\operatorname{dim} \mathscr{H}_{1}=2$ (the other cases being similar) so that

$$
T_{1}:=\left(\begin{array}{cc}
\lambda & 1 \\
0 & \lambda
\end{array}\right), \quad P_{\lambda, 1}:=\frac{1}{2 \pi i} \oint_{\Gamma_{\lambda}}\left(z-T_{1}\right)^{-1} d z .
$$

where $\Gamma_{\lambda}$ is (for example) the circle of center $\lambda$ and radius 1 . Let $n \in \mathbb{N}$. Recall that

$$
\frac{1}{2 \pi i} \oint_{\Gamma_{\lambda}}(z-\lambda)^{-n} d z=\frac{1}{2 \pi i} \oint_{\theta=0}^{2 \pi} e^{i(1-n) \theta} d \theta=\left\{\begin{array}{lll}
1 & \text { if } & n=1 \\
0 & \text { if } & n \neq 1
\end{array}\right.
$$

It follows that

$$
P_{\lambda, 1}:=\frac{1}{2 i \pi} \oint_{\Gamma_{\lambda}}\left(\begin{array}{cc}
(z-\lambda)^{-1} & -(z-\lambda)^{-2} \\
0 & (z-\lambda)^{-1}
\end{array}\right) d z=\left(\begin{array}{ll}
1 & 0 \\
0 & 1
\end{array}\right)=\operatorname{Id}_{\mathscr{H}_{1}} .
$$

The application $P_{\lambda}$ is indeed the projection on $\mathscr{H}_{1}$.

Corollary 2.13. If $\lambda \in \mathrm{Sp}(\mathrm{T})$ is isolated with a finite algebraic multiplicity, then it is necessarily an eigenvalue. 


\section{Analytic Matrix Functions}

\section{1 n-Dimensional perturbation theory}

Let $X(t)$ be a complex valued $n \times n$ matrix function defined on a real interval $I$.

The following results are taken from $[2,13,14]$ where proofs and more results on this topic can be found.

Definition 3.1. When we say that $X(t)$ is analytic in a neighborhood of $t=t_{0}$, we mean that each element of $X(t)$ is representable as a Taylor series centered at $t_{0}$ which converges in some neighborhood of $t_{0}$.

Example 3.2. Consider the matrix valued analytic matrix

$$
X(\beta)=\left[\begin{array}{cc}
1 & \beta \\
\beta & -1
\end{array}\right]
$$

Its eigenvalues are

$$
\lambda_{ \pm}(\beta)= \pm \sqrt{\beta^{2}+1} .
$$

We notice the following features:

1. $X(\beta)$ is an entire function but the eigenvalues are not entire (they have singularities as functions of $\beta$ ); the singularities are not on the real axis where $X(\beta)$ is self-adjoint but occur at non real $\beta$, namely at $\beta= \pm i$.

2. at the singular values of $\beta$, that is, at $\beta= \pm i$ there are fewer distinct eigenvalues, namely one, than at other points where there are two.

3. at the singular values of $\beta$ the matrix $X(\beta)$ is not diagonalizable.

If $X(\beta)$ is a matrix-valued analytic function in a connected region $R$ of the complex plane, then the eigenvalues of $X(\beta)$ are solutions of the equation

$$
\operatorname{det}(X(\beta)-\lambda I)=(-1)^{n}\left(\lambda^{n}+a_{1}(\beta) \lambda^{n-1}+\cdots+a_{n}(\beta)=0 .\right.
$$

Theorem 3.3. Let

$$
F(\beta, \lambda)=\lambda^{n}+a_{1}(\beta) \lambda^{n-1}+\cdots+a_{n}(\beta)
$$

be a polynomial of degree $n$ in $\lambda$ whose leading coefficient is one and whose other coefficients are all analytic functions of $\beta$.

1. Suppose that $\lambda=\lambda_{0}$ is a simple root of $F\left(\beta_{0}, \lambda\right)$. Then for $\beta$ near $\beta_{0}$, there is exactly one root $\lambda(\beta)$ of $F(\beta, \lambda)$ near $\lambda_{0}$, and $\lambda(\beta)$ is analytic in $\beta$ near $\beta=\beta_{0}$.

2. Suppose that $\lambda=\lambda_{0}$ is a root of multiplicity $m$ of $F\left(\beta_{0}, \lambda\right)$. Then for $\beta$ near $\beta_{0}$, there are exactly $m$ roots (counting multiplicities) of $F(\beta, \lambda)$ near $\beta_{0}$, and these roots are branches of one or more multivalued analytic functions with at most algebraic points at $\beta=\beta_{0}$. Explicitly, there exist $p_{1}, \cdots, p_{k}$ with $\sum_{i=1}^{k} p_{i}=m$ and multivalued analytic functions $\lambda_{1}, \cdots, \lambda_{k}$ (not necessarily distinct) with convergent Puiseux series

$$
\lambda_{i}(\beta)=\lambda_{0}+\sum_{j=1}^{\infty} \alpha_{j}\left(\beta-\beta_{0}\right)^{\frac{j}{p}}
$$

so that the $m$ roots near $\lambda_{0}$ are given by the $p_{1}$ values of $\lambda_{1}$, the $p_{2}$ values of $\lambda_{2}, \cdots$, etc.

Corollary 3.4. Let $X(\beta)$ be a matrix-valued analytic function near $\beta_{0}$.

1. If $\lambda_{0}$ is a simple root of $X\left(\beta_{0}\right)$, then for $\beta$ near $\beta_{0}, X(\beta)$ has exactly one root $\lambda_{0}(\beta)$ near $\lambda_{0} ;\left(\lambda_{0}(\beta)\right.$ is a simple and analytic eigenvalue if $\beta$ is near $\beta=\beta_{0}$.

2. If $\lambda_{0}$ is an eigenvalue of $T\left(\beta_{0}\right)$ of algebraic multiplicity $m$, then for $\beta$ near $\beta_{0}, X(\beta)$ has exactly m eigenvalues (counting multiplicity) near $\lambda_{0}$. These eigenvalues are all the branches of one or more multivalued functions analytic near $\beta_{0}$ with at most algebraic singularities at $\beta_{0}$. 


\subsection{Analytic perturbation of self-adjoint and hermitian matrices}

If $X$ and $Y$ are self-adjoint, the perturbed eigenvalues of $X+\beta Y$ are analytic at $\beta=0$ even if $X$ has degenerate eigenvalues. That the branch points allowed by the last theorem do not occur in this case is a theorem of F. Rellich in [14]. The example at the beginning of this section shows that the branch points can occur for non real $\beta$ even in the self-adjont case, $X(\beta)^{*}=X(\bar{\beta})$.

Theorem 3.5 ([14]). Suppose that $X(\beta)$ is a matrix-valued analytic function in a region $R$ containing a section of the real axis, and that $X(\beta)$ is self-adjoint for $\beta$ real. Let $\lambda_{0}$ be an eigenvalue of $X\left(\beta_{0}\right)$ of multiplicity $m$. If $\beta_{0}$ is real, there are distinct functions $\lambda_{1}(\beta), \cdots, \lambda_{p}(\beta)$, single-valued and analytic in a neighborhood of $\beta_{0}$, which are all the eigenvalues.

For analytic Hermitian matrices the next explicit formulation of Theorem 4 holds.

Theorem 3.6 ([14]). Let $X(\lambda)$ be an $n \times n$ Hermitian matrix function which is analytic on a real interval $(a, b)$ in which $\operatorname{det} X(\lambda) \neq 0$. There exists an analytic unitary matrix function $U(\lambda), \lambda \in(a, b)$ and analytic real-valued functions $\mu_{i}(\lambda), \lambda \in$ $(a, b)$, such that

$$
X(\lambda)=U(\lambda)\left[\begin{array}{ccccc}
\mu_{1}(\lambda) & 0 & \cdots & \cdots & 0 \\
0 & \mu_{2}(\lambda) & \cdots & \cdots & 0 \\
\vdots & \vdots & \ddots & \vdots & \vdots \\
0 & 0 & \cdots & \ddots & 0 \\
0 & 0 & \cdots & 0 & \mu_{n}(\lambda)
\end{array}\right] U(\lambda)^{-1}
$$

Remark 3.7. The interesting aspect of this theorem is its validity even at points where the multiplicity of the eigenvalues changes. When $X(\lambda)$ is not hermitian its eigenvalues usually have branch points. Moreover, [14] contains a counterexample which shows that the theorem cannot be extended to infinitely differentiable, but not holomorphic functions. In that counterexample, $X(\lambda)$ and the eigenvalues are still everywhere differentiable, but all eigenvectors are discontinuous at the point $\lambda=0$. It will be seen later that the sharpest results, such as in [7] for hermitian matrices, are obtained when we deal with conditions close to the ones satisfied in F. Rellich [14]. Otherwise, Example 1 and the previous discussion show some of the difficulties we encounter when we consider general analytic matrix-valued functions (and similarly for operator-valued functions), which force us to impose extra conditions, like the constancy on the number of eigenvalues or constancy on the number of Jordan blocks and other similar conditions (see [6] for examples and a more complete list of references on matrices commuting with their derivatives).

\subsection{Analytic matrix functions commuting with their derivatives}

We start with a result on analytic matrices which commute with their derivatives.

Theorem 3.8 ([15]). Let $X(t)$ be an $n \times n$ analytic matrix function defined on a real interval $I$. Suppose that for all $t \in I$, the eigenvalues of $X(t)$ are distinct, and $\left[X(t), X^{\prime}(t)\right]=0$ on I. Then $[X(s), X(t)]=0$ for all $s \neq t$ in $I$.

Proof. If for $t \in I$, eigenvalues of $X(t)$ are distinct, then there exists a constant invertible matrix $U$ such that

$$
X(t)=U D(t) U^{-1},
$$

where $D(t)$ is diagonal. Thus

$$
[X(s), X(t)]=0
$$

for $s \neq t$ in $I$.

Remark 3.9. The paper [15] contains examples showing that the conclusion fails to hold if the eigenvalues of $X(t)$ are not all distinct. Jean Dieudonné proved in [4] that Theorem 3.8 holds if $X(t)$ is only continuously differentiable.

Next we recall an important theorem contained in [6].

Theorem 3.10 ([6]). If $X$ is an analytic matrix function, defined on an open interval $\Lambda$ of $\mathbb{R}$, taking its values in $\mathscr{M}_{n}$ and commuting with its derivative on $\Lambda$, and if $t_{0} \in \Lambda$ and $\mu_{1}, \cdots, \mu_{p}$, are the distinct eigenvalues of $X\left(t_{0}\right)$ of respective algebraic multiplicities $m_{1}, \cdots, m_{p}$, then on a neighborhood $\Lambda_{t_{0}} \cap \Lambda$ of $t_{0}, A$ has the form

$$
X(t)=U \operatorname{diag}\left(\boldsymbol{m}_{1}(t), \cdots, \boldsymbol{m}_{p}(t)\right) U^{-1}
$$

where $t \in \Lambda_{t_{0}}, U$ is an invertible matrix, and for each $k \in\{1,2, \cdots, p\}, \boldsymbol{m}_{K}$ is an analytic matrix function from $\Lambda_{t_{0}}$ into $\mathscr{M}_{m_{k}}$ commuting with its derivative on $\Lambda_{t_{0}}$, such that $\operatorname{Sp}\left(\boldsymbol{m}_{k}\left(t_{0}\right)\right)=\left\{\mu_{k}\right\}$. 


\subsubsection{Diagonalizable matrix functions}

As in [6], we say that a matrix $X$ from a set $\Lambda$ into $\mathscr{M}_{n}$ is pointwise diagonalizable on $\Lambda$ if, for every $t \in \Lambda$, there exists an invertible matrix $U(t)$ such that

$$
U(t)^{-1} X(t) U(t)
$$

is diagonal, whereas we say that $A$ is globally diagonalizable on $\Lambda$ if there exists an invertible matrix $U$ such that, for all $t \in \Lambda$, the matrix

$$
U^{-1} X(t) U
$$

is diagonal.

A family $(X(t))_{t \in \Lambda}$ of matrices of $\mathscr{M}_{n}$ is functionally commutative if

$$
[X(t), X(s)]=0
$$

for all $s, t \in \Lambda$.

Theorem 3.11 ([7]). If $(X(s))$ is an analytic family of hermitian matrices such that $\left[X(s), X^{\prime}(s)\right]=0$ for all $s$ in an interval I of $\mathbb{R}$, then the family $(X(s))$ is commutative, i.e. $[X(s), X(t)]=0$ for all $s, t \in I$.

Proof. It is well known that $X(t)=\sum_{i=1}^{r} \lambda_{i}(t) G_{i}$, where the $G_{i}$ are projection matrices such that $G_{i}^{2}=G_{i}$ and $G_{i} G_{j}=G_{j} G_{i}=0$ if $i \neq j$. Now $\left[X(t), X^{\prime}(t)\right]=0$ yields that $G_{i}$ is constant for each $i$. Hence,

$$
X(s) X(t)=\sum_{i=1}^{r} \lambda_{i}(s) \lambda_{i}(t) G_{i}=X(t) X(s) .
$$

Remark 3.12. The condition of analyticity cannot be relaxed in Theorem 8 . Indeed, let $X$ and $Y$ two $n \times n$ constant noncommutative hermitian matrices and define $A(t)=X \exp \left(-t^{2}\right)$ if $t<0, A(0)=0, A(t)=Y \exp \left(-t^{2}\right)$ if $t>0$. Then $A(t)$ is hermitian, of class $\mathscr{C}^{\infty}$, and not analytic, and commutes with its derivative on $\mathbb{R}$. However if $s<0$ and $t>0$, then $A(s) A(t) \neq A(t) A(s)$.

The next theorem is a generalization of Theorem 3.8 to continuously differentiable matrices commuting with their derivatives.

Theorem $3.13([6])$. If $(X(s))$ is a continuously differentiable family of diagonalizable matrices with a constant number of eigenvalues, such that

$$
\left[X(s), X^{\prime}(s)\right]=0
$$

for all $s$ in an interval I of $\mathbb{R}$, then the family $(X(s))$ is functionally commutative, i.e.

$$
[X(s), X(t)]=0
$$

for all $s, t \in I$.

Question 1. Is it possible to extend the previous theorem to the case of an analytic family $(X(s))$ of matrices (without the condition of diagonalizability)?

If we replace 'differentiable' by 'analytic' and remove 'constant number of eigenvalues' in Theorem 3.13, we obtain the following result.

Theorem 3.14. If $X(s)$ is an analytic family of diagonalizable matrices, such that $\left[X(s), X^{\prime}(s)\right]=0$ for all $s$ in a real interval $I$, then $X(s)$ is functionally commutative on $I \backslash S$ where $S$ is an exceptional set containing a finite number of points, i.e. $[X(s), X(t)]=0$ for all $s, t \in I \backslash S$.

Proof. Follows from Theorem 3.4.25 in [1]. 


\subsection{Characterization of functional commutativity}

Definition 3.15. An analytic matrix function $X$ defined on a contour $\Gamma$ is said to be functionnally commutative if $[X(s), X(t)]=0$ whenever $s, t \in \Gamma$.

It is natural to try to express matrix-valued functions which commute with their derivative in their simplest form possible. The following lemma and theorem are adapted from [3], where similar results were proven for measurable functions.

Lemma 3.16. The matrix function $G$ is functionally commutative if and only if

$$
G(t)=\sum_{j=1}^{m} \phi_{j}(t) G_{j}
$$

where $m \leq n^{2}$ and $G_{j}$ are pairwise commuting constant matrices and $\phi_{j}$ are analytic scalar functions.

Proof. $(\Leftarrow)$ The sufficiency of the conditions of the lemma is evident.

$(\Rightarrow)$ Let $\mathscr{L}$ be the linear hull of the set of all matrices of the form $G(t), t \in \Gamma$ in the space of all constant matrices of $n$th order and choose in it a basis $G_{1}, \ldots, G_{m}$. Since the space of $n \times n$ matrices has dimension $n^{2}$, then $m \leq n^{2}$. Furthermore $G\left(t_{1}\right) G\left(t_{2}\right)=G\left(t_{2}\right) G\left(t_{1}\right)$ implies that any two matrices from $\mathscr{L}$ commute. Consequently, all matrices $G_{1}, \ldots, G_{m}$ commute pairwise. Finally, as $G(t) \in \mathscr{L}$ for all $t \in \Gamma$ and $\left\{G_{j}\right\}_{j=1}^{m}$ is a basis in $\mathscr{L}$, then there exists a unique representation $G(t)$ in the form of a linear combination of the $G_{j}$ 's. Supposing $\phi_{j}(t)$ to be equal to the $j$ th coefficient of this linear combination, we have $G(t)=\sum \phi_{j}(t) G_{j}$

Next, we seek some relations between the entries of matrix-valued functions which are functionally commutative. To do so, let us answer the simple question: what is functional commutativity of two by two matrices?

Theorem 3.17. The matrix function $G=\left(\begin{array}{ll}g_{11} & g_{12} \\ g_{21} & g_{22}\end{array}\right)$ is functionally commutative if and only if $g_{11}-g_{22}, g_{12}$ and $g_{21}$ are scalar multiples of one and the same function $\phi$, i.e. $g_{12}(t)=\alpha \phi(t), g_{21}(t)=\beta \phi(t), g_{11}(t)-g_{22}(t)=\gamma \phi(t)$ where $\alpha, \beta, \gamma$ are numbers.

Proof. $(\Leftarrow)$ If the conditions

$$
g_{12}(t)=\alpha \phi(t), g_{21}(t)=\beta \phi(t), g_{11}(t)-g_{22}(t)=\gamma \phi(t)
$$

are fulfilled, then

$$
G(t)=\left(\begin{array}{cc}
g_{22}(t)+\gamma \phi(t) & \alpha \phi(t \\
\beta \phi(t & g_{22}(t)
\end{array}\right) .
$$

A direct verification shows that, for any $s, t \in \Gamma$, we have

$$
G(s) G(t)=G(t) G(s) .
$$

$(\Rightarrow)$ let the matrix function $G$ be functionally commutative. At first, suppose that $g_{11}(t)=g_{22}(t)$. If in addition, $g_{12}=$ $g_{21}=0$, then the conditions

$$
g_{12}(t)=\alpha \phi(t), g_{21}(t)=\beta \phi(t), g_{11}(t)-g_{22}(t)=\gamma \phi(t)
$$

are satisfied for $\alpha=0, \beta=0, \gamma=0$.

In the opposite case, a point $t_{0} \in \Gamma$ can be found such that at least one of the functions $g_{12}$ or $g_{21}$ is different from zero. Equating the diagonal elements of the matrices $G(t) G\left(t_{0}\right)$ and $G\left(t_{0}\right) G(t)$, we get $g_{12}(t) g_{21}\left(t_{0}\right)=g_{12}\left(t_{0}\right) g_{21}(t)$. If $g_{21}\left(t_{0}\right) \neq 0$, we may take $\phi=g_{21}, \beta=1, \gamma=\frac{g_{12}\left(t_{0}\right)}{g_{21}\left(t_{0}\right)}$, and if $g_{12}\left(t_{0}\right) \neq 0$, then we can choose $\phi=g_{12}, \alpha=1, \gamma=0, \beta=\frac{g_{21}\left(t_{0}\right)}{g_{12}\left(t_{0}\right)}$. Now, let the functions $g_{11}$ and $g_{22}$ be different. Then there exists a point $t_{0} \in \Gamma$ such that $g_{11}\left(t_{0}\right) \neq g_{22}\left(t_{0}\right)$. Equating the elements outside the diagonals of the matrices $G(t) G\left(t_{0}\right)$ and $G\left(t_{0}\right) G(t)$, we obtain

$$
\left(g_{11}(t)-g_{22}(t)\right)\left(g_{12}\left(t_{0}\right)\right)=\left(g_{11}\left(t_{0}\right)-g_{22}\left(t_{0}\right)\right)\left(g_{12}(t)\right),
$$

and

$$
\left(g_{11}(t)-g_{22}(t)\right)\left(g_{21}\left(t_{0}\right)\right)=\left(g_{11}\left(t_{0}\right)-g_{22}\left(t_{0}\right)\right)\left(g_{21}(t)\right),
$$

so that we may set

$$
\phi=g_{11}-g_{22}, \gamma=1, \alpha=\frac{g_{12}\left(t_{0}\right)}{\left(g_{11}\left(t_{0}\right)-g_{22}\left(t_{0}\right)\right)}, \beta=\frac{g_{21}\left(t_{0}\right)}{\left(g_{11}\left(t_{0}\right)-g_{22}\left(t_{0}\right)\right)},
$$

and the theorem is proved. 


\section{Extension to Infinite-Dimensional Case}

\subsection{Spectral theorem for normal compact operators}

We recall that $\mathscr{B}(\mathscr{H})$ denotes the Banach algebra of all bounded operators on the complex Hilbert space $\mathscr{H}$ and by definition the spectrum of $T$, denoted by $\operatorname{Sp}(T)$, is the set of $\lambda \in \mathbb{C}$ such that $T-\lambda I$ is not invertible in $\mathscr{B}(\mathscr{H})$. We recall the important theorem on the spectrum of a compact operator on a Hilbert space.

Theorem 4.1. Let $T \subset \mathscr{B}(\mathscr{H})$ be compact. Then $\mathrm{Sp}(\mathrm{T})$ is a non-empty compact subset of $\mathbb{C}$ with no accumulation point other than zero. Each non-zero $\lambda \in \mathrm{Sp}(T)$ is an isolated eigenvalue of $T$ with finite algebraic multiplicity.

Theorem 4.2 (Spectral theorem for compact operators). If an operator $T \in \mathscr{B}[\mathscr{H}]$ on a non-zero Hilbert space $\mathscr{H}$ is compact and normal, then there exists a unique countable resolution of the identity $\left\{E_{k}\right\}$ on $\mathscr{H}$ and a bounded set of scalars $\lambda_{k}$ for which $T=\sum_{k=1}^{\infty} \lambda_{k} E_{k}$, where $\left\{\lambda_{k}\right\}=\operatorname{Sp}_{p}(T)$ is the (non-empty) set of all (distinct) eigenvalues of $T$ and each $E_{k}$ is the orthogonal projection onto the eigenspace $\mathscr{N}\left(\lambda_{k} I-T\right)$ (i.e., $\mathscr{R}\left(E_{k}\right)=\mathscr{N}\left(\lambda_{k} I-T\right)$ ). If the above countable weighted sum of projections is infinite, then it converges in the (uniform) topology of $\mathscr{B}(\mathscr{H})$.

Proof. See [11], Theorem 3.3 on page 58.

Remark 4.3. It is worth mentioning here that according to Proposition 4.K in [11], the projections $E_{k}$ coincide with the Riesz projections associated to spectral values $\lambda_{k}$ as usually obtained by the Holomorphic Functional Calculus.

Definition 4.4. An operator-valued function $T: \Omega \mapsto \mathscr{B}(\mathscr{H})$, defined on an open subset $\Omega$ of the complex plane $\mathbb{C}$, is said to be analytic at $z_{0} \in \Omega$ if there are operators $T_{n} \in \mathscr{B}(\mathscr{H})$ and a positive number $\delta$ such that

$$
T(z)=\sum_{n=0}^{\infty}\left(z-z_{0}\right)^{n} T_{n},
$$

where the power series on the right-hand side converges with respect to the operator norm on $\mathscr{B}(\mathscr{H})$ in a disc $\left|z-z_{0}\right|<\delta$ for some $\delta>0$. We say that $T$ is analytic or holomorphic in $\Omega$ if it is analytic at every point in $\Omega$.

\subsection{Riesz projections depending on a parameter}

Let $A(t)$ denote an analytic family of normal compact operators on a complex Hilbert space and defined on a real interval $I=(a, b)$ with $a<0<b$. Let $\lambda$ be an eigenvalue of $A(0)$. More generally $A(t)$ can be an analytic family of bounded normal operators on $\mathscr{H}$ with the property that $\lambda$ is an isolated point of the spectrum $\operatorname{Sp} A(0)$, and such that the $\lambda$-eigenspace of $A(0)$ is finite-dimensional. Let $D$ be a closed disk centered at $\lambda$ such that $\operatorname{Sp} A(0) \cap D=\{\lambda\}$. It follows that, for $t$ sufficiently small, $\operatorname{Sp} A(t) \cap \gamma=\emptyset$ where $\gamma=\partial D$ is the boundary of $D$. For such $t$, we have the orthogonal Riesz projections

$$
P_{\lambda}(t)=\frac{1}{2 \pi i} \int_{\gamma}(\xi I-A(t))^{-1} d \xi
$$

with range $\mathscr{H}(t)$, depending analytically on $t$, such that $P(0)$ is the orthogonal projection of $\mathscr{H}$ onto the $\lambda$-eigenspace of $A(0)$. Our main result will be based essentially on the possibility of decomposing an operator like $A(t)$ into a sum

$$
\sum_{k=1}^{\infty} \mu_{k} P_{k}(t)
$$

where the $P_{k}(t)$ are mutually orthogonal analytic projections, such that

$$
\left[A(t), P_{k}(t)\right]=0 .
$$

It is a spectral decomposition with the added condition of analyticity. For more details on the spectrum, riesz idempotents/projections, spectral decomposition of operators and related questions to spectral theory see [1], [10],[11], [13]. 


\subsection{Analytic family of compact operators on a Hilbert space}

Our goal is to extend Theorem 3.11 from matrices to compact operators on a Hilbert space. We proved in [12] the following theorem for self-adjoint compact operators.

Theorem $4.5([12])$. If $(A(s))$ is an analytic family of compact self-adjoint operators on a Hilbert space, such that $\left[A(s), A^{\prime}(s)\right]=$ 0 for all $s$ in an interval I of $\mathbb{R}$, then $(A(s))$ is functionally commutative, i.e. $[A(s), A(t)]=0$ for all $s, t \in I$.

Thanks to Theorem 3.3 of [11] we can extend our previous theorem to compact normal operators on a Hilbert space.

Theorem 4.6. If $(A(s))$ is an analytic family of normal compact operators on a Hilbert space, such that $\left[A(s), A^{\prime}(s)\right]=0$ for all $s$ in an interval $I$ of $\mathbb{R}$, then $(A(s))$ is functionally commutative, i.e. $[A(s), A(t)]=0$ for all $s, t \in I$.

\subsection{Analytic normal compact operators on a Hilbert space commuting with their derivatives}

The following results may be proved in much the same way as their equivalent ones in [12].

Lemma 4.7. Let $A(t)$ be an analytic family of normal compact operators on a Hilbert space $\mathscr{H}$ which commute with its derivative. Then the projections associated to the eigenvalues of $A(t)$ commute with their derivative.

Proof. Similar to the proof of Lemma 2 in [12] if we replace self-adjoint by normal.

Lemma 4.8. If a family of projections $P(t)$ commutes with its derivative on an interval $I \subset \mathbb{R}$, then $P(t)$ is constant.

Proof. Similar to the proof of Lemma 3 in [12] if we replace self-adjoint by normal.

Theorem 4.9. Let $A(t)$ be an analytic family of normal compact operators on a Hilbert space $H$. Suppose that $A(t)$ commutes with its derivative for all $t \in I \subset \mathbb{R}$. Then $A(t)$ is functionally commutative, i.e. $[A(s), A(t)]=0$ for all $s, t \in I$.

Proof. Similar to the proof of Theorem 2 in [12] if we replace self-adjoint by normal.

It remains to solve the following two more general extensions by dropping either compactness of the operators or normality.

Problem 4.10. If $(A(s))$ is an analytic family of compact operators, without quasi-nilpotent component (this is the case for self-adjoint operators on a Hilbert space), on a Banach space, such that $\left[A(s), A^{\prime}(s)\right]=0$ for all $s$ in an interval I of $\mathbb{R}$, then $(A(s))$ is functionally commutative, i.e. $[A(s), A(t)]=0$ for all $s, t \in I$.

Problem 4.11. If $(A(s))$ is an analytic family of self-adjoint operators on a Hilbert space, such that $\left[A(s), A^{\prime}(s)\right]=0$ for all $s$ in an interval $I$ of $\mathbb{R}$, then the family $(A(s))$ is functionally commutative, i.e. $[A(s), A(t)]=0$ for all $s, t \in I$.

Final comments. In analytic perturbation theory, we are concerned with the analytic dependence of various quantities on the parameter $x$, assuming that the given family $T(x)$ of operators is analytic. Among the quantities that have been considered so far, there are the resolvent $R_{\lambda}(T)=(\lambda-T)^{-1}$, the isolated eigenvalues $\lambda_{n}$ and the associated spectral eigenprojections or Riesz idempotents. The general form of the spectral theorem ([11], Theorem 3.15) for self-adjoint operators furnishes other functions to be considered. One of them is the spectral family $E(\lambda, x)$ for $T(x)$, defined for real $x$, where $T(x)$ is a self-adjoint family (see [10] for more details). This combined with Theorem 3.15 in [11], might perhaps be the path to explore towards a possible solution of the second problem.

\section{References}

[1] B. Aupetit, A Primer On Spectral Theory, Universitext, Springer-Verlag, 1991.

${ }^{[2]}$ H. Baumgartel, Analytic Perturbation Theory for Matrices and Operators, Operator Theory: Advances and Applications, 15, Birkhäuser, 1985.

${ }^{[3]}$ K. Clancy, I. Gohberg, Factorization of matrix functions and singular integral operators, Oper. Theory Adv. Appl., 3, Birkhäuser Verlag (Basel) 1981.

[4] J. Dieudonné, Sur un théorème de Schwertfeger, Ann. Polon. Math. 24(1974), 87 - 88.

[5] J.C. Evard, Conditions for a vector subspace $E(t)$ and for a projector $P(t)$ not to depend on $t$. , Lin. Alg. Appl. 91(1987), 121-131.

[6] J. C. Evard, On matrix functions which commute with their derivative, Lin. Alg. Appl. 68(1985), 145 - 178.

[7] S. Goff, Hermitian function matrices which commute with their derivative, Lin. Alg. Appl. 36(1981), 33 - 40. 


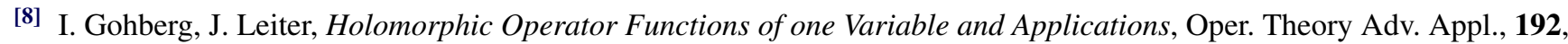
2009.

[9] I. Gohberg, S. Goldberg, M.A. Kaashoek, Classes of linear operators, 1, Birkhauser 1990.

[10] T. Kato, Perturbation Theory for Linear Operators, Springer, 1980.

[11] C.S. Kubrusly, Spectral Theory of Bounded Linear Operators, Birkhauser 2020.

[12] A. Maouche, Functional commutativity of analytic families of self adjoint compact operators on a Hilbert space, Commun. Adv. Math. Sci., 3(1) (2020), 9 - 12.

[13] M. Reed, B. Simon, Modern Methods of Mathematical Physics, Academic Press, 1975.

[14] F. Rellich, Perturbation Theory of Eigenvalue Problems, Institute of Mathematical Sciences, New York, 1950.

${ }^{[15]}$ H. Schwertfeger, Sur les matrices permutables avec leur derivée, Rend. Sem. Mat. Univ. Politec. Torino. 11(1952), 329 333. 